\title{
Quantum-classical approximation beyond Redfield theory
}

\author{
A. A. Neufeld ${ }^{\text {a) }}$ \\ Abteilung Spektroskopie und Photochemische Kinetik, Max-Planck-Institut für Biophysikalische Chemie, \\ Am Fassberg, D-37077 Göttingen, Germany
}

(Received 9 January 2003; accepted 6 May 2003)

\begin{abstract}
A quantum-classical approximation, capable of describing the evolution of open quantum systems well beyond the applicability limits of Redfield theory is suggested. The theory is based on the short lifetime of the quantum correlations between the quantum and the classical subsystem, caused by energy dispersion (per degree of freedom) of the canonical bath. The resulting quantum-classical approximation has the form of two auxiliary differential equations and fully accounts both for the arbitrary long memory of the heat reservoir and detailed balance. These equations allow direct solution in the time domain without constructing/diagonalizing Liouville space operators, and, in combination with molecular dynamics techniques to simulate bath dynamics, may be applied to quantum subsystems with a fairly large number of levels. A simple example of a two-level system, coupled to a single correlation time canonical bath, was considered to demonstrate different regimes of approaching the canonical equilibrium state. (C) 2003 American Institute of Physics.
\end{abstract}

[DOI: $10.1063 / 1.1587124]$

\section{INTRODUCTION}

A consistent treatment of condensed phase reactions has a great importance for chemistry, physics, and biology. Due to the complexity of such systems, where interactions between reactants and the surrounding molecules cannot be neglected, the kinetics and dynamics of elementary reactions in liquids/dense gases still is not completely understood. Unlike reactions in rarefied gases, liquid state processes are affected by complicated many-body interactions, resulting in cage effect, solvent-induced vibrational energy relaxation, etc.

The fully quantum-mechanical treatment of many-body systems is inaccessible, as the computational cost grows exponentially with the number of degrees of freedom. However, in many cases the reacting systems in condensed phase may be represented by a relatively small subset of quantum modes (usually the internal states of the reactants, the reaction coordinates) and a thermal reservoir (bath). The theory of open quantum systems, coupled to a dissipative environment, is being developed by many research groups.

There exist a variety of approaches to open quantum systems, ranging from rigorous methods with known applicability limits (Redfield theory ${ }^{1}$ and its extensions, ${ }^{2-5}$ nonMarkovian theories, ${ }^{6-12}$ Golden-Rule perturbation approach, ${ }^{13}$ forward-backward initial value representation method $^{14}$ ) to some semiempirical schemes (surface hopping, ${ }^{15}$ time-dependent self-consistent field, ${ }^{16,17} \mathrm{Car}-$ Parrinello density functional, ${ }^{18}$ Gaussian wave packet ${ }^{19}$ approaches). The choice between them depends on the phenomenon under consideration and the characteristic parameter values.

In the present article we mainly focus on vibrational energy relaxation (VER) of molecules in solutions and dense gases. This problem puts quite specific demands on the the-

${ }^{a)}$ Electronic mail: aneufel@gwdg.de oretical approach. First, a finite temperature of the bath has to be taken into account, since the splittings between vibrational energy levels are often comparable to the mean energy of heat reservoir and, thus, an equilibrium (relaxed) state exhibits considerable population differences. Besides, the lack of the detailed balance principle in the description of VER leads to continuous heating of the vibrational degrees of freedom, as the energy spectrum is unrestricted from above. Second, VER is very sensitive to the character of motion of the surrounding molecules, and the approach should allow for realistic modeling of the bath dynamics. Usually VER processes occur in the picosecond time range, and classical molecular dynamics (MD) seems to be most suitable for the $a b$ initio simulation of the bath at moderate to long time scales and at sufficiently high temperatures to avoid quantum effects in the dynamics of the heat reservoir.

Up to now only Redfield theory, modified to account for the finite temperature of the bath, seems to meet the demands. An appealing feature of the Redfield approach is that it allows a straightforward modification to incorporate a classical description of the bath dynamics, see Ref. 5, for instance. Nevertheless, the Redfield approach is based on the Markov approximation with respect to memory effects and, therefore, has rather narrow applicability limits, concerning both the allowed strength of the system-bath interactions and the time resolution. Recently there appeared several nonMarkovian theories, suitable to describe a short-time evolution of the quantum subsystem, which, however, either omit the detailed balance principle or represent the bath as a set of uncoupled harmonic oscillators. The latter assumption is reasonable when studying ultrafast processes in liquids, as on very short time scales the motion of molecules in solutions mainly consists of their vibrations. However, both the anharmonicity of bath modes and the coupling between them seem to be important characteristics of the bath dynamics at longer times. Thus, there is no reliable method to simulate VER 
processes beyond the limitations of Redfield theory, and the present article attempts to fill up this gap.

The outline of the article is as follows: The small parameter of the theory, its physical sense, and the applicability limits are discussed in Sec. II. The closed integrodifferential master equation for the evolution of the reduced density matrix of the quantum-dynamical subsystem (QDS) is obtained in Sec. III. Although the general procedure of the derivation is well known, see for example Ref. 4, we repeat it here for self-consistency and to ensure that the approximations made are compatible with the desired applicability range. The memory kernel is explicitely evaluated in Sec. IV, assuming the bilinear form of the QDS-bath coupling Hamiltonian. This assumption, however, was made only to present the results in a more compact form, while the quantum-classical formulation, see Sec. V, remains in force for a general form of the QDS-bath interaction as well.

The application of non-Markovian theories to simulate the kinetics of VER is rather difficult. Usually the linear integrodifferential equations are solved via the Fourier/ Laplace transform technique. Unfortunately, it requires the inversion of the Liouville space operator and, therefore, is computationally expensive even for QDSs with moderate number of the states. However, if the bath is treated classically, the derived non-Markovian master equation can be reduced to two auxiliary differential equations without narrowing the applicability limits, see Sec. V.

Basic properties of the density matrix (conservation of the trace, hermiticity, positivity) are discussed in Sec. VI in connection to the suggested non-Markovian quantumclassical approximation (NQCA). A simple model of the two level system (as the QDS), interacting with a single correlation time canonical bath, was used to demonstrate basic features of the relaxation kinetics towards to the equilibrium state, Sec. VII. A more realistic description of the bath via molecular dynamics technique and in the frameworks of the developed NQCA will be applied in subsequent article. The main results and their possible applications are summarized in Conclusion.

\section{GENERAL REMARKS}

Strictly speaking, any physically justified separation of the QDS and the bath requires their weak correlation. Mathematically this is equivalent to the smallness of the corresponding off-diagonal elements in the total (QDS+ bath) density matrix $\varrho$. These elements in the basis of the direct product of the QDS and the bath states are of the form,

$$
\varrho_{i k}^{\alpha \beta} \equiv\langle\alpha|\langle i|\varrho| k\rangle| \beta\rangle, \quad \alpha \neq \beta, \quad i \neq k,
$$

where Latin and Greek letters label the QDS and the bath states, respectively. Indeed, the elements $\varrho_{i k}^{\alpha \alpha}$ describe the correlations inside the QDS, while $\varrho_{i i}^{\alpha \beta}$ correspond to the correlations between bath states. The specific mechanism providing weak QDS-bath correlations depends on the system under consideration. The most widely used assumptions, however, are the weak coupling limit and the fast decay of correlations due to bath dynamics. In the former case, the matrix elements of the QDS-bath interactions $\hat{W}$ are as-

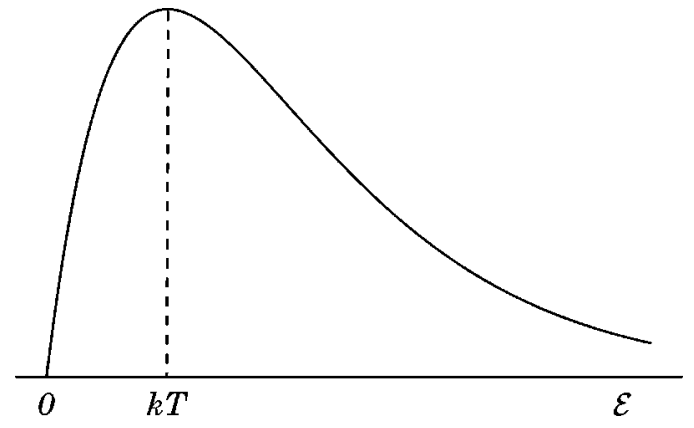

FIG. 1. Energy distribution per degree of freedom in the Gibbs canonical ensemble.

sumed to be small compared to the splitting between corresponding levels, while in the latter case one requires

$$
\left|W_{i k}^{\alpha \beta}\right| \tau_{b} / \hbar \ll 1, \quad \alpha \neq \beta, \quad i \neq k,
$$

where $\tau_{b}$ is a characteristic lifetime of the QDS-bath correlations. None the less, both approaches actually utilize the weak correlation of the QDS and the bath.

It is often believed, see Refs. 20, 21, for instance, that the lifetime of the QDS-bath correlations $\tau_{b}$ is of order of the correlation time of the heat reservoir $\tau_{c}$, i.e., defined by the bath dynamics. Usually the correlation time for the rotational motion of molecules and their fragments in solutions lies in the picosecond time range, while for the translational motion (cage effect) $\tau_{c}$ may fall into nanoseconds. However, such correlations have purely "classical" character, they are readily determined via the two-dimensional conditional probability density $\mathcal{P}\left(q, t \mid q_{0}, t_{0}\right)$ of generalized set of coordinates $q$ at time $t$, being $q_{0}$ at the initial instant of time $t_{0}$. In other words, the "classical" correlations correspond to the correlations between bath states, i.e., to the elements $\varrho_{i i}^{\alpha \beta}$ in the fully quantum-mechanical picture. On the other hand, the factorization of the total density matrix into the direct product of the density matrices of the QDS and the bath requires their weak cross-correlations (1). In the following, we shall call them "quantum" correlations as they do not have classical counterparts.

The decay of the quantum correlations depends strongly on the statistical properties of the bath. In particular, if the bath is well described by the Gibbs canonical (constant temperature) ensemble, there exists an efficient mechanism leading to the decay of the QDS-bath cross-correlations. Indeed, the distribution of energies $(\mathcal{E})$ per bath degree of freedom in the canonical ensemble is proportional to $\mathcal{E} \exp \left(-\mathcal{E} / k_{B} T\right)$, where $k_{B}$ and $T$ are the Boltzmann constant and the absolute temperature, correspondingly, and is plotted schematically in Fig. 1. The maximum of the distribution is located at $\mathcal{E}$ $=k_{B} T$, and the mean square deviation of the energy is $k_{B} T$ as well.

Since the energies of the bath degrees of freedom have an uncertainty of $k_{B} T$, the quantum correlations decay with a characteristic time $\tau_{b} \sim \hbar /\left(k_{B} T\right)$ due to the phase cancellation at longer times. Thus, averaging over canonical ensemble prior to the actual simulations provides fast decay of the quantum correlations, at room temperature $\hbar /\left(k_{B} T\right)$ $\sim 10 \mathrm{fs}$, which is much shorter than the typical classical cor- 
relation times. This is in contrast to the approaches, where averaging over the bath is taken after propagation in time (wave function based methods, etc.). The qualitative difference between the methods can be demonstrated by some analog from classical mechanics. Many processes, affected by the stochastic motion of molecules in liquids/dense gases may be modeled either via the Fokker-Planck equation or by molecular dynamics methods. In the former case, averaging over the stochastic process is done at the stage of the derivation of the equation, while in the MD approach simulations are performed along deterministic trajectories with the subsequent averaging of the results. The Fokker-Planck equation contains the correlation time parameter, which can be used to develop certain approximations (Markov limit, for instance). On the other hand, the correlation time parameter does not exist for the single MD trajectory, as it characterizes the statistical ensemble, but not the single trajectory.

The theory developed in the present article is based on assumption (2), where $\tau_{b}$ is the lifetime of the quantum correlations provided by the energy dispersion of the bath degrees of freedom. Note, that $\tau_{b}$ is not a relaxation time of the bath, but characterizes its statistical properties. The relaxation requires energy flow between the bath degrees of freedom, and, therefore, depends on the coupling of the bath modes. On the other hand, energy dispersion of the degrees of freedom is a characteristics of the bath in the equilibrium, i.e., when all relaxation processes are finished, that is why $\tau_{b}$ is an universal function of temperature. In the following we assume that the bath is not affected by the QDS evolution, though there are many situations when the feedback of the QDS evolution on the dynamics of the classical degrees of freedom can not be neglected. Examples are charge transfer in polar media, conformational changes of molecules, etc. The full feedback between the quantum and the classical degrees of freedom will be considered elsewhere.

\section{GENERAL QUANTUM MASTER EQUATION}

The density matrix formalism is the most consistent way to describe a QDS interacting with a thermal bath. The starting point is the Liouville-von Neumann equation for the total density matrix $\varrho(t)$,

$$
\frac{d \varrho(t)}{d t}=-\frac{i}{\hbar}[\hat{H}+\hat{F}+\hat{W}, \varrho(t)], \quad \varrho(0)=\varrho_{0},
$$

where $\hat{H}, \hat{F}$, and $\hat{W}$ are the Hamiltonians of the QDS, bath and QDS-bath coupling, respectively. In the following we set $\hbar=1$, which corresponds to the common practice of working in frequency units. Besides, we also set the Boltzmann constant $k_{B}=1$ and measure the temperature in frequency units as well. Then, one has $1 \mathrm{~K}=k_{B} / \hbar=1.31$ $\times 10^{11} \mathrm{rad} / \mathrm{s}$.

From now on, it is convenient to formulate the problem in Liouville space. ${ }^{22}$ It greatly reduces intermediate derivations and allows to present results in a compact form. Moreover (although this is not used in the present article), the Liouville formalism makes it possible to include nonHamiltonian dynamics (like spontaneous radiative decay of the states) at no extra cost. Nevertheless, all equations of practical interest are finally transformed back into the original Hilbert space representation.

In the Liouville representation Eq. (3) takes the form,

$$
\frac{d \bar{\varrho}(t)}{d t}=i(\hat{\mathcal{H}}+\hat{\mathcal{F}}+\hat{\mathcal{W}}) \bar{\varrho}(t)+\delta(t) \varrho_{0},
$$

where $\hat{\mathcal{H}}, \hat{\mathcal{F}}$, and $\hat{\mathcal{W}}$ are the Liouville operators, denoted by the corresponding calligraphic symbols. The relations between matrix elements of the operators in the Hilbert and the Liouville spaces are given in Appendix A. Here we also extended the definition of the density matrix for negative times using the stepwise Heaviside $\theta$-function, i.e.,

$$
\bar{\varrho}(t)=\theta(t) \varrho(t) .
$$

Thus, $\bar{\varrho}(t)$ is a generalized function of time and allows for a two-sided Fourier transform. The theory of the generalized functions and their properties can be found in Ref. 23.

The Liouville-von Neumann equation (3) or (4) is not well suited to develop approximations and, therefore, is transformed into an equivalent integrodifferential one as follows. The Fourier transform of Eq. (4) gives

$$
(i \omega-i \hat{\mathcal{H}}-i \hat{\mathcal{F}}) \tilde{\varrho}(\omega)-i \hat{\mathcal{W}} \tilde{\varrho}(\omega)=\varrho_{0},
$$

where

$$
\tilde{\varrho}(\omega)=\int_{-\infty}^{\infty} \bar{\varrho}(t) e^{-i \omega t} d t
$$

Introducing the free resolvent $\hat{\mathcal{G}}_{0}(\omega)$,

$$
(i \omega-i \hat{\mathcal{H}}-i \hat{\mathcal{F}}) \hat{\widetilde{\mathcal{G}}}_{0}(\omega)=\hat{1},
$$

which describes evolution of the QDS and the bath in the absence of their coupling, we rewrite Eq. (6) in the following form:

$$
\tilde{\varrho}(\omega)=\hat{\widetilde{\mathcal{G}}}_{0}(\omega) \varrho_{0}+\hat{\widetilde{G}}_{0} i \hat{\mathcal{W}} \tilde{\varrho}(\omega) .
$$

The proof of Eq. (9) is evident, it is sufficient to act on both sides of Eq. (9) by $(i \omega-i \hat{\mathcal{H}}-i \hat{\mathcal{F}})$, and from Eq. (8) we immediately come to Eq. (6).

Substituting Eq. (9) into the last term on the left-hand side of Eq. (6), and taking the inverse Fourier transform, we get the exact integrodifferential equation, fully equivalent to the original Liouville-von Neumann Eq. (4). It takes the form,

$$
\begin{aligned}
\frac{d \bar{\varrho}(t)}{d t}= & \left(\delta(t)+i \hat{\mathcal{W}} \hat{\mathcal{G}}_{0}(t)\right) \varrho_{0}+i(\hat{\mathcal{H}}+\hat{\mathcal{F}}) \bar{\varrho}(t) \\
& -\int_{-\infty}^{\infty} \hat{\mathcal{K}}(\tau) \bar{\varrho}(t-\tau) d \tau,
\end{aligned}
$$

where

$$
\hat{\mathcal{K}}(\tau)=\hat{\mathcal{W}} \hat{\mathcal{G}}_{0}(\tau) \hat{\mathcal{W}}, \quad \hat{\mathcal{G}}_{0}(\tau)=\theta(\tau) e^{i \hat{\mathcal{H}} \tau} e^{i \hat{\mathcal{F}} \tau} .
$$

Bath variables are present in Eq. (10), but the kernel $\hat{\mathcal{K}}(\tau)$ is known explicitly. This is in contrast to the NakajimaZwanzig generalized master equation, ${ }^{24}$ where the bath variables are excluded at the expense of a complicated structure 
of the memory kernel. The required perturbation expansion over weak QDS-bath cross-correlations to their lowest order is easy to make using the factorized density matrix of the form (we neglect the feedback of the QDS evolution on the dynamics of the bath degrees of freedom)

$$
\bar{\varrho}(t-\tau)=\bar{\sigma}(t-\tau) \times \rho_{b}
$$

in the integrand on the right hand side of Eq. (10). Here,

$$
\rho_{b}=\frac{e^{-\hat{F} / T}}{Z_{b}}, \quad Z_{b}=\operatorname{Tr}_{b}\left(e^{-\hat{F} / T}\right)
$$

is the equilibrium density matrix of the canonical bath $\left(\operatorname{Tr}_{b}\right.$ denotes the trace over the bath degrees of freedom). Then we get the closed integrodifferential equation for the reduced density matrix of the QDS, which is obtained from Eq. (10) by taking the trace over the bath states to give

$$
\begin{aligned}
\frac{d \bar{\sigma}(t)}{d t}= & \delta(t) \sigma_{0}+\bar{\zeta}(t)-i[\hat{H}, \bar{\sigma}(t)] \\
& -\int_{-\infty}^{\infty} \hat{\mathcal{M}}(\tau) \bar{\sigma}(t-\tau) d \tau
\end{aligned}
$$

where

$$
\begin{aligned}
& \bar{\sigma}(t)=\operatorname{Tr}_{b} \bar{\varrho}(t), \quad \sigma_{0}=\operatorname{Tr}_{b} \varrho_{0}, \\
& \bar{\zeta}(t)=\operatorname{Tr}_{b}\left(i \hat{\mathcal{W}} \hat{\mathcal{G}}_{0}(t) \varrho_{0}\right),
\end{aligned}
$$

and the reduced memory kernel

$$
\hat{\mathcal{M}}(\tau)=\operatorname{Tr}_{b}\left(\hat{\mathcal{K}}(\tau) \rho_{b}\right)=\operatorname{Tr}_{b}\left(\hat{\mathcal{W}} \hat{\mathcal{G}}_{0}(\tau) \hat{\mathcal{W}} \rho_{b}\right)
$$

Despite the fact, that the factorized density matrix (12) was used to obtain the closed integrodifferential equation (14) for the QDS evolution, the quantum correlations between the QDS and the bath are taken into account perturbatively. Indeed, the cross-correlations between the QDS and the bath appear due to the interactions between them and are generally strictly proportional to the quadratic power of the interaction. But the exact kernel in Eq. (10) is already proportional to the second power of the coupling, and, therefore, the effect of the cross-correlations on the dynamics of QDS is retained in Eq. (14) up to their lowest order.

The right-hand side of Eq. (14) contains both an instant source term due to the initial conditions and the timeextended source $\bar{\zeta}(t)$. Potential renormalization is required to provide fast decay of the additional source. Indeed, the free propagator $\hat{\mathcal{G}}_{0}(t)$ has the following property

$$
\hat{\mathcal{G}}_{0}(t) \varrho_{0} \underset{t \gg \tau_{b}}{\simeq} e^{i \hat{\mathcal{H}} t} \sigma_{0} \times \rho_{b},
$$

which leads to

$$
\bar{\zeta}(t) \underset{t \gg \tau_{b}}{\simeq} \operatorname{Tr}_{b}\left(i \hat{\mathcal{W}} \rho_{b}\right) e^{i \hat{\mathcal{H}} t} \sigma_{0}
$$

Thus, the renormalization of the form,

$$
\hat{\mathcal{H}} \rightarrow \hat{\mathcal{H}}+\operatorname{Tr}_{b}\left(\hat{\mathcal{W}} \rho_{b}\right), \quad \hat{\mathcal{W}} \rightarrow \hat{\mathcal{W}}-\operatorname{Tr}_{b}\left(\hat{\mathcal{W}} \rho_{b}\right),
$$

where the trace is taken only over the bath variables, eliminates the source term on a time scale larger than the quantum correlations lifetime $\tau_{b}$. In the following we assume that the renormalization is done, i.e., that

$$
\operatorname{Tr}_{b}\left(\hat{\mathcal{W}} \rho_{b}\right)=0
$$

Then, the source term $\bar{\zeta}(t)$ is nonzero only in the presence of the initial correlations between the QDS and the bath and then rapidly decays at times exceeding $\tau_{b}$. We would emphasize, however, that the renormalization concerns only the diagonal over bath variables part of the QDS-bath coupling, which does not induce transitions between bath states.

The derived non-Markovian master equation (14) for the reduced density matrix can be solved via Fourier transform method, which yields algebraic equation of the form,

$$
i \omega \widetilde{\sigma}(\omega)+i[\hat{H}, \widetilde{\sigma}(\omega)]+\hat{\mathcal{M}}(\omega) \widetilde{\sigma}(\omega)=\sigma_{0}+\widetilde{\zeta}(\omega),
$$

and provided that the Fourier transform of the memory kernel and of the source term,

$$
\begin{aligned}
& \hat{\tilde{\mathcal{M}}}(\omega)=\int_{-\infty}^{\infty} \hat{\mathcal{M}}(t) e^{-i \omega t} d t, \\
& \tilde{\zeta}(\omega)=\int_{-\infty}^{\infty} \bar{\zeta}(t) e^{-i \omega t} d t
\end{aligned}
$$

are known. Here

$$
\widetilde{\sigma}(\omega)=\int_{-\infty}^{\infty} \bar{\sigma}(t) e^{-i \omega t} d t
$$

is the Fourier transform of the reduced density matrix.

Nonetheless, the detailed information about energy levels of the bath, necessary to determine the bath propagator $e^{i \hat{\mathcal{F}} \tau}$, is not available for realistic heat reservoirs, especially in the thermodynamic limit. In reality, the information about dynamics of the heat reservoir enters either via the spectral density of the bath or via the autocorrelation function of the QDS-bath coupling. An explicit evaluation of the memory kernel (16) in a form suitable to introduce quantum-classical treatment of the bath is given in the next section.

\section{EVALUATION OF MEMORY KERNEL}

The explicit evaluation of the memory kernel (16) is done assuming a simple (factorized) form of the QDS-bath coupling Hamiltonian $\hat{W}$, when it can be written as a product of two operators which have matrix elements only in the QDS or the bath subspaces, i.e.,

$$
\hat{W}=\hat{V} \hat{B}, \quad \hat{W}_{i k}^{\alpha \beta}=\hat{V}_{i k} \hat{B}^{\alpha \beta} .
$$

However, the above assumption is made only to provide a transparent derivation of the kernel structure, while the main results of the article, see Sec. V, remain valid for the general form of $\hat{W}$ as well.

Taking into account a bilinear form of the QDS-bath coupling Hamiltonian (24) and successively applying Eqs. (A9) and (A13), we derive from Eq. (16) the following expression for the integral term on the right-hand side of Eq. (14), 


$$
\begin{aligned}
\int_{-\infty}^{\infty} \hat{\mathcal{M}}(\tau) \bar{\sigma}(t-\tau) d \tau \\
=\int_{-\infty}^{\infty} \theta(\tau)\left(\left[\hat{V}, e^{-i \hat{H} \tau} \hat{V} \bar{\sigma}(t-\tau) e^{i \hat{H} \tau}\right] j_{1}(\tau)\right. \\
\left.\quad-\left[\hat{V}, e^{-i \hat{H} \tau} \bar{\sigma}(t-\tau) \hat{V} e^{i \hat{H} \tau}\right] j_{2}(\tau)\right) d \tau,
\end{aligned}
$$

where $j_{1,2}(\tau)$ have the sense of the "quantum" correlation functions, and are defined as

$$
\begin{aligned}
& j_{1}(\tau)=\frac{1}{Z_{b}} \operatorname{Tr}_{b}\left(\hat{B}_{h}(\tau) \hat{B}_{h}(0) e^{-\hat{F} / T}\right), \\
& j_{2}(\tau)=\frac{1}{Z_{b}} \operatorname{Tr}_{b}\left(\hat{B}_{h}(0) \hat{B}_{h}(\tau) e^{-\hat{F} / T}\right) .
\end{aligned}
$$

Here

$$
\hat{B}_{h}(\tau)=e^{i \hat{F} \tau} \hat{B} e^{-i \hat{F} \tau}
$$

is the bath operator in the Heisenberg representation.

The quantum correlation functions are not independent, but related to each other. First,

$$
j_{2}(\tau)=j_{1}(-\tau) .
$$

The second relation is established using the two-sided Fourier transform, i.e., when we consider the entire time axis, including negative times. It gives ${ }^{25}$

$$
\widetilde{j}_{2}(\omega)=e^{\omega / T} \widetilde{j}_{1}(\omega),
$$

where

$$
\tilde{j}_{1,2}(\omega)=\int_{-\infty}^{\infty} j_{1,2}(\tau) e^{-i \omega \tau} d \tau
$$

Mathematical proofs of Eqs. (28) and (29) are given in Appendix B. The established relation (29) allows to rewrite the Fourier transform of Eq. (25) solely via $\tilde{j}_{1}(\omega)$. The resulting expression takes the form,

$$
\begin{aligned}
\hat{\tilde{\mathcal{M}}}(\omega) \widetilde{\sigma}(\omega)= & \sum_{k, m}\left(\left[\hat{V}, \hat{P}^{k} \hat{V} \widetilde{\sigma}(\omega) \hat{P}^{m}\right]-\left[\hat{V}, \hat{P}^{k} \widetilde{\sigma}(\omega) \hat{V} \hat{P}^{m}\right]\right. \\
& \left.\times e^{\left(\omega+\omega_{k m}\right) / T}\right) \tilde{j}_{1}\left(\omega+\omega_{k m}+i \varepsilon\right), \quad \varepsilon \rightarrow+0 .
\end{aligned}
$$

Here we used the following identity for the operator exponent:

$$
e^{ \pm i \hat{H} \tau} \equiv \sum_{n} \hat{P}^{n} e^{ \pm i E_{n} \tau}
$$

where $\hat{P}^{n}$ are the projector operators on the eigenstates of the QDS Hamiltonian, while $E_{n}$ are the corresponding eigenvalues, i.e.,

$$
\hat{P}^{n}=|n\rangle\langle n|, \quad \hat{H}| n\rangle=E_{n}|n\rangle, \quad \omega_{k m} \equiv E_{k}-E_{m} .
$$

In principle, Eqs. (21) and (31) are sufficient to describe the kinetics of the QDS relaxation towards to its Boltzmann equilibrium, if $\widetilde{j}_{1}(\omega)$ is known. For the spin-boson model, and when the spectral density of the bath has smooth ohmic form with frequency cutoff $\omega_{c},{ }^{26}$ it is of the form

$$
\widetilde{j}_{1}(\omega)=\frac{2 \pi \eta \omega e^{-|\omega| / \omega_{c}}}{1-e^{-\omega / T}},
$$

where $\eta$ is a phenomenological friction coefficient. However, in case the bath is treated classically, e.g., by molecular dynamics methods, the information about $\widetilde{j}_{1}(\omega)$ is not immediately available, since the quantum correlation functions $j_{1,2}(\tau)$, defined by Eqs. (26), do not have classical analogs. Indeed, the quantum correlation functions are defined on the entire time axis (including negative times), and are the averages of the corresponding time-ordered products of the bath operators. This is in contrast to the classical correlation functions, which are defined from a given moment of time, providing the retarded or the advanced evolution. Besides, in the classical limit bath operators commute with each other, and, therefore, the time-ordered averages (26) do not have classical counterparts.

Nevertheless, the memory kernel can be rewritten using quantities which may be directly identified with the classical correlation functions. For this purpose we introduce the symmetrized correlation function $\mathcal{J}(\tau)$ along the entire time axis,

$$
\mathcal{J}(\tau)=\left(j_{1}(\tau)+j_{2}(\tau)\right) / 2, \quad \mathcal{J}(-\tau)=\mathcal{J}(\tau),
$$

where the last property follows from Eq. (28), and the corresponding retarded and advanced correlation functions,

$$
\overline{\mathcal{J}}_{\text {ret }}(\tau)=\theta(\tau) \mathcal{J}(\tau), \quad \overline{\mathcal{J}}_{\text {adv }}(\tau)=\theta(-\tau) \mathcal{J}(\tau),
$$

which possess all necessary properties to be identified with the classical correlation functions. The Fourier transform of Eq. (35) gives

$$
\begin{aligned}
& \tilde{\mathcal{J}}(\omega)=\left(1+e^{\omega / T}\right) \tilde{j}_{1}(\omega) / 2, \\
& \widetilde{\mathcal{J}}(\omega)=\int_{-\infty}^{\infty} \mathcal{J}(\tau) e^{-i \omega \tau} d \tau,
\end{aligned}
$$

where we have also used Eq. (29). Thus, the Fourier transform of the quantum correlation function $\tilde{j}_{1}(\omega)$ is expressed via the Fourier transform of the symmetrized correlation function $\widetilde{\mathcal{J}}(\omega)$ as

$$
\widetilde{j}_{1}(\omega)=\frac{2 \widetilde{\mathcal{J}}(\omega)}{1+e^{\omega / T}} .
$$

Substituting Eq. (38) into Eq. (31) we obtain

$$
\begin{aligned}
\hat{\mathcal{M}}(\omega) \widetilde{\sigma}(\omega)= & \sum_{k, m}\left(\left[\hat{V}, \hat{P}^{k}[\hat{V}, \widetilde{\sigma}(\omega)] \hat{P}^{m}\right]-\tanh \left(\frac{\omega+\omega_{k m}}{2 T}\right)\right. \\
& \left.\times\left[\hat{V}, \hat{P}^{k}[\hat{V}, \widetilde{\sigma}(\omega)]_{+} \hat{P}^{m}\right]\right) \tilde{\mathcal{J}}\left(\omega+\omega_{k m}+i \varepsilon\right),
\end{aligned}
$$

where $[\ldots, \ldots]_{+}$denotes anticommutator.

The derived non-Markovian master equation for the reduced density matrix of the QDS, given by Eqs. (21) and (39), is based on the quantum-mechanical description both of the QDS and the bath. However, unlike Eq. (31), it allows for classical treatment of the bath degrees of freedom. In that case the Fourier transform of the symmetrized correlation function is replaced by its classical analog, ${ }^{27}$ i.e., 


$$
\widetilde{\mathcal{J}}(\omega)=\widetilde{\mathcal{J}}_{\text {ret }}(\omega)+\widetilde{\mathcal{J}}_{\text {adv }}(\omega) \rightarrow \widetilde{\mathcal{J}}_{\text {ret }}^{\text {classical }}(\omega)+\widetilde{\mathcal{J}}_{\text {adv }}^{\text {classical }}(\omega)
$$

It is a matter of common knowledge, that all mixed quantum-classical treatments use some uncontrolled approximations and are not equivalent. Thus, the formulation of the quantum-classical approximation presents a big challenge. However, the identification of the symmetrized correlation function with the sum of classical correlation functions (40) seems to be a quite natural way to introduce classical description of the bath degrees of freedom. Finally, Eqs. (21), (39) can be used if the bath is treated both quantummechanically and classically. In the former case, the Fourier transform of the symmetrized correlation function is expressed via the known Fourier transform of the quantum correlation function $\tilde{j}_{1}(\omega)$ using Eq. (37), while in the latter case it is replaced by the sum of Fourier transforms of the classical correlation functions (40).

The derived Eq. (39) effectively decouples the statistical and the dynamical properties of the heat reservoir. The former enter via universal (dynamics independent) function of the temperature, while the information about bath dynamics is contained in the Fourier transform of the symmetrized correlation function $\widetilde{\mathcal{J}}(\omega)$ which, however, implicitly depends on the temperature as well. Thus, the classical treatment is introduced only to describe the dynamical properties of the heat reservoir, while the statistical properties of the bath are taken into account quantum-mechanically.

\section{DIFFERENTIAL FORM OF THE QUANTUM-CLASSICAL APPROXIMATION}

Although the non-Markovian master equation (14) can be directly solved by the Fourier transform technique, it is not convenient for practical purposes. First of all, in order to solve Eq. (21) one requires to find the inverse Liouville space operator, and the unfavorable scaling (the dimensionality of the Liouville space operators is $N^{2} \times N^{2}, N$ is the number of the states in the QDS) makes it possible only for rather small systems. Besides, the solution has to be found for many distinct values of the Fourier transform parameter $\omega$, for the subsequent use in the inverse transform to restore the kinetics.

The reduction of the non-Markovian master equation to a Markovian one usually calls for some additional approximations and can be done in several ways. The most popular approximation utilizes fast decay (on the time scale $t \sim \tau_{c}$, where $\tau_{c}$ is the characteristic correlation time) of the bath correlation function, and requires weak effect of the QDSbath interactions on the time scale of $\tau_{c}$. Then, the following approximation:

$$
\bar{\sigma}(t-\tau) \simeq e^{-i \hat{\mathcal{H}} \tau} \bar{\sigma}(t)=e^{i \hat{H} \tau} \bar{\sigma}(t) e^{-i \hat{H} \tau}
$$

can be made in the integral term of Eq. (14), which result in the Markovian master equation [contribution from the initial correlations $\bar{\zeta}(t)$ can be neglected for large times as well] of the form,

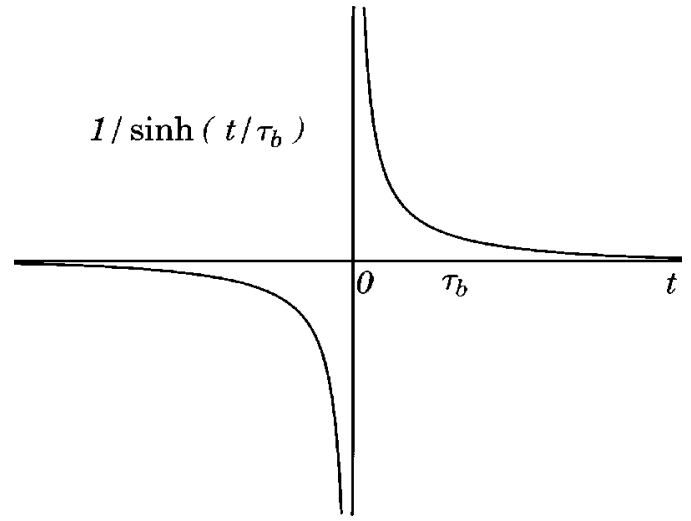

FIG. 2. Time behavior of the generalized kernel $f_{b}(t)$, defined by Eq. (43). It has singularity $\sim 1 / t$ at $t \rightarrow 0$ and decays exponentially at $t \rightarrow \pm \infty$.

$$
\begin{aligned}
& \frac{d \bar{\sigma}(t)}{d t}=\delta(t) \sigma_{0}-i[\hat{H}, \bar{\sigma}(t)]-\hat{\mathcal{R}} \bar{\sigma}(t), \\
& \hat{\mathcal{R}}=\int_{-\infty}^{\infty} \hat{\mathcal{M}}(\tau) e^{-i \hat{\mathcal{H}} \tau} d \tau, \quad t \gg \tau_{c},
\end{aligned}
$$

well known as the Redfield theory. It can be directly solved in the time domain, and, besides, the explicit construction of the full Redfield tensor $\hat{\mathcal{R}}$ can be avoided, ${ }^{5}$ which allows application to a fairly large QDSs. The deficiency of the Redfield approach concerns its rather severe restrictions, both for the allowed strength of the system-bath interactions and for the applicability time scale $\left(t \gg \tau_{c}\right)$. Many processes in condensed phase, however, occur in a subpicosecond time period, while the shortest (rotational) classical correlation times are usually about ten picoseconds. The non-Markovian master equation, Eqs. (21) and (31), allows for a larger strength of the QDS-bath interaction and is valid for shorter times as well. In the case, when the bath is treated classically, it can be reduced to the differential form without narrowing the applicability limits.

As it was pointed out in Sec. IV, Eq. (39) effectively decouples the statistical and the dynamical properties of the heat reservoir. The information about the statistical properties enters via dynamics independent function of the temperature, which has the following forms in the time and the Fourier domains,

$$
\begin{aligned}
& f_{b}(t)=\frac{1}{\pi \tau_{b}} \frac{1}{\sinh \left(t / \tau_{b}\right)}, \quad \tau_{b}=\frac{1}{\pi T}, \\
& \widetilde{f}_{b}(\omega)=i \tanh \left(\frac{\omega}{2 T}\right) .
\end{aligned}
$$

The generalized memory kernel $f_{b}(t)$, defined by Eq. (43), has a singularity at $t=0$ and sharply decays at longer times with the characteristic time $\tau_{b}$, see Fig. 2. It describes the decay of the QDS-bath cross-correlations at the expense of the phase cancellation process due to the energy dispersion of the degrees of freedom of the canonical bath. The characteristic time $\tau_{b}$ was estimated in Sec. II to be $\sim 1 / T$ ( $\hbar$ $=k_{B}=1$ ), while the accurate expression (43) yields an even shorter lifetime of the quantum correlations. At room temperature $(T=300 \mathrm{~K})$, one has $\tau_{b}=8 \mathrm{fs}$. 
Then, the first term in the right-hand side of Eq. (39) can be interpreted as a high temperature limit, when the energy dispersion of the bath degrees of freedom is so large, that the quantum correlations decay instantly (the Fourier transform of $\delta$-function is constant, and is independent on $\omega$ ). The second term in the right-hand side of Eq. (39) accounts for the finite lifetime of the quantum correlations, and the functional form of $f_{b}(t)$ is similar to the derivative of $\delta$-function, but has a finite width of $\tau_{b}$.

The high temperature limit term describes irreversible evolution, while the other term in the right-hand side of Eq. (39) accounts for the reversibility of the dynamics on the time scale $\tau_{b}$ of the quantum correlations decay. Indeed, only the Fourier transform of the retarded correlation function contributes to the high temperature part of the memory kernel $\hat{\mathcal{M}}(t>0)$, since the Fourier transform of the advanced correlation function does not have singularities in the upper plane of the complex surface. The situation is quite different for the other term in Eq. (39), as $\widetilde{f}_{b}(\omega)$ has own singularities in the upper plane at $\omega_{n}=2 \pi i(n+1 / 2) T,(n=0,1,2, \ldots)$ and both the Fourier transform of the retarded and the advanced correlation functions contribute to the inverse Fourier transform in the vicinities of $\omega_{n}$.

The assumption of the weak cross-correlations between the QDS and the bath, employed in the present article, requires that the matrix elements of the QDS-bath interaction satisfy the condition,

$$
\left|\hat{W}_{i k}^{\alpha \beta}\right| \tau_{b}=\frac{\left|\hat{W}_{i k}^{\alpha \beta}\right|}{\pi T} \ll 1, \quad i \neq k, \quad \alpha \neq \beta,
$$

where the temperature and the QDS-bath coupling are in frequency units. But it also means, that the evolution of the density matrix $\bar{\sigma}(t)$ is weakly affected by the QDS-bath interaction on the time scale of the quantum correlations decay $\tau_{b}$, and the main effect of the QDS-bath coupling takes place at times $t \gg \tau_{b}$. Then, the second term on the righthand side of Eq. (39) can be further simplified as follows. First, we use the identity,

$$
\begin{aligned}
\widetilde{f}_{b}\left(\omega+\omega_{k m}\right)\left[\hat{V}, \hat{P}^{k}[\hat{V}, \widetilde{\sigma}(\omega)]_{+} \hat{P}^{m}\right] \\
=\int_{-\infty}^{\infty} e^{-i \omega t} \int_{-\infty}^{\infty} f_{b}(\tau)\left[\hat{V}, \hat{P}^{k} e^{-i \hat{H} \tau}\right. \\
\left.\quad \times[\hat{V}, \bar{\sigma}(t-\tau)]_{+} e^{i \hat{H} \tau} \hat{P}^{m}\right] d \tau d t,
\end{aligned}
$$

and expand $\bar{\sigma}(t-\tau)$ in the vicinity of $\tau \sim 0$ using the fast decay of the kernel $f_{b}(\tau)$. Note, that the off-diagonal elements of the density matrix may evolve fast compared to $1 / \tau_{b}$, since the splittings between QDS levels can be arbitrary. Hence, we expand the density matrix in the interaction representation $\bar{\sigma}_{i}$, which is related to $\bar{\sigma}$ as

$$
\bar{\sigma}_{i}(t)=e^{i \hat{H} t} \bar{\sigma}(t) e^{-i \hat{H} t}, \quad \bar{\sigma}(t)=e^{-i \hat{H} t} \bar{\sigma}_{i}(t) e^{i \hat{H} t},
$$

to get

$$
\begin{aligned}
\bar{\sigma}(t-\tau) & \simeq e^{\tau \sim \tau_{b}} \\
& =e^{i \hat{H} \tau(t-\tau)}\left(\bar{\sigma}(t)-i[\hat{H}, \bar{\sigma}(t)] \tau-\frac{\left.d \bar{\sigma}_{i}(t)-\tau \frac{d}{d t}\right)}{d t} \tau\right) e^{i \hat{H}(t-\tau)}
\end{aligned}
$$

The obtained expansion is similar to Eq. (41), but is more accurate. The role of the additional terms in Eq. (47), which account for an early non-Markovian evolution of the density matrix, is explained later in this article.

Substitution of Eq. (47) in the right-hand side of Eq. (45) eliminates convolution, which gives

$$
\begin{aligned}
\tilde{f}_{b}\left(\omega+\omega_{k m}\right)\left[\hat{V}, \hat{P}^{k}[\hat{V}, \widetilde{\sigma}(\omega)]_{+} \hat{P}^{m}\right] \\
\simeq\left[\hat{V}, \hat{P}^{k}\left([\hat{U}, \widetilde{\sigma}(\omega)]_{+}\right.\right. \\
\left.\left.\quad+[\hat{Y}, i[\hat{H}, \widetilde{\sigma}(\omega)]+i \omega \widetilde{\sigma}(\omega)]_{+}\right) \hat{P}^{m}\right],
\end{aligned}
$$

where

$$
\hat{U}=\int_{-\infty}^{\infty} f_{b}(\tau) e^{-i \hat{H} \tau} \hat{V} e^{i \hat{H} \tau} d \tau=i \sum_{k, m} \tanh \left(\frac{\omega_{k m}}{2 T}\right) \hat{P}^{k} \hat{V} \hat{P}^{m},
$$

and

$$
\hat{Y}=-\int_{-\infty}^{\infty} \tau f_{b}(\tau) e^{-i \hat{H} \tau} \hat{V} e^{i \hat{H} \tau} d \tau=\frac{1}{2 T} \sum_{k, m} \frac{\hat{P}^{k} \hat{V} \hat{P}^{m}}{\cosh ^{2}\left(\frac{\omega_{k m}}{2 T}\right)} .
$$

Thus, Eq. (39) can be simplified to give

$$
\begin{aligned}
\hat{\tilde{\mathcal{M}}}(\omega) \widetilde{\sigma}(\omega) \simeq & \sum_{k, m}\left[\hat{V}, \hat{P}^{k}\left([\hat{V}, \widetilde{\sigma}(\omega)]+i[\hat{U}, \widetilde{\sigma}(\omega)]_{+}\right.\right. \\
& +i[\hat{Y}, i[\hat{H}, \widetilde{\sigma}(\omega)] \\
& \left.\left.+i \omega \widetilde{\sigma}(\omega)]_{+}\right) \hat{P}^{m}\right] \widetilde{\mathcal{J}}_{\text {ret }}\left(\omega+\omega_{k m}\right),
\end{aligned}
$$

which serves as a basis for the subsequent considerations. Here we replaced the Fourier transform of the symmetrized correlation function by the Fourier transform of the retarded correlation function, see discussion above Eq. (44). In other words, on the time scale $t \gg \tau_{b}$ the dynamics becomes irreversible, which is related to the decay of the quantum correlations.

Sometimes memory-type equations can be reduced to a set of auxiliary differential equations. For a spin-boson model and exponentially decaying kernel an equivalent set of differential equations was obtained in Refs. 7, 8. Below we derive differential form of NQCA for a more general case. In the classical limit Eq. (24) takes the form,

$$
\hat{W}(q)=\hat{V} B(q), \quad \int B^{2}(q) d q=1,
$$

where the normalization condition was introduced for the sake of conveniency, while the correlation function of the bath operators is determined via the two-dimensional probability density $\mathcal{P}\left(q, q_{0} ; \tau\right)$ of generalized coordinate $q$ at time $\tau$, given coordinate $q_{0}$ at the initial instant of time. Here and below $q$ denotes the generalized set of coordinates, specify- 
ing the state of the system in its phase space, e.g., it may involve momenta of motion as well. Then, the classical correlation function is defined as

$$
\overline{\mathcal{J}}_{\text {ret }}(\tau)=\iint B(q) \overline{\mathcal{P}}\left(q, q_{0} ; \tau\right) B\left(q_{0}\right) d q d q_{0},
$$

where we extended the definition of $\mathcal{P}\left(q, q_{0} ; \tau\right)$ to negative times via the Heaviside $\theta$-function, i.e.,

$$
\overline{\mathcal{P}}\left(q, q_{0} ; \tau\right)=\theta(\tau) \mathcal{P}\left(q, q_{0} ; \tau\right) .
$$

In turn, the two-dimensional probability density $\overline{\mathcal{P}}\left(q, q_{0} ; \tau\right)$ satisfies the classical equation of motion of the form

$$
\frac{\partial}{\partial t} \overline{\mathcal{P}}\left(q, q_{0} ; \tau\right)=\mathcal{L}_{q} \overline{\mathcal{P}}\left(q, q_{0} ; \tau\right)+\delta(t) \delta\left(q-q_{0}\right),
$$

where $\mathcal{L}_{q}$ is the linear functional operator, defining the evolution of the system in its phase space. In particular, for the simplest case, corresponding to stochastic jumps of infinite length, one has

$$
\mathcal{L}_{q}=1 / \tau_{c}, \quad \overline{\mathcal{P}}\left(q, q_{0} ; t\right)=\theta(t) e^{-t / \tau_{c}} \delta\left(q-q_{0}\right) .
$$

Here $\tau_{c}$ is the characteristic time between the jumps. [For the stochastic jumps of finite length there always exists a diffusional tail at long times, and the "infinite" means that we restrict ourselves to the exponential part in $\overline{\mathcal{P}}\left(q, q_{0} ; t\right)$.]

Then Eqs. (21) and (51) are equivalent in the time domain to the following set of differential equations,

$$
\frac{d \bar{\sigma}(t)}{d t}=\delta(t) \sigma_{0}+\bar{\zeta}(t)-i[\hat{H}, \bar{\sigma}(t)]-i \int[\hat{W}(q), \bar{\eta}(q, t)] d q,
$$

$$
\begin{aligned}
\frac{\partial \bar{\eta}(q, t)}{\partial t}= & -i[\hat{H}, \bar{\eta}(q, t)]-i[\hat{W}(q), \bar{\sigma}(t)]+[\hat{U}(q), \bar{\sigma}(t)]_{+} \\
& +\left[\hat{Y}(q), i[\hat{H}, \bar{\sigma}(t)]+\frac{d \bar{\sigma}(t)}{d t}\right]_{+}+\mathcal{L}_{q} \bar{\eta}(q, t),
\end{aligned}
$$

where $\bar{\eta}(t)$ is an auxiliary matrix with the same dimensionality as the QDS density matrix $\bar{\sigma}(t)$, and

$$
\begin{aligned}
& \hat{U}(q)=i \sum_{k, m} \tanh \left(\frac{\omega_{k m}}{2 T}\right) \hat{P}^{k} \hat{W}(q) \hat{P}^{m}, \\
& \hat{Y}(q)=\frac{1}{2 T} \sum_{k, m} \frac{\hat{P}^{k} \hat{W}(q) \hat{P}^{m}}{\cosh ^{2}\left(\frac{\omega_{k m}}{2 T}\right)},
\end{aligned}
$$

see also Eqs. (49) and (50). The mathematical proof of the equivalence of Eqs. (57), (58), and (21), (51), where the retarded correlation function is identified with its classical analog (53), is given in Appendix C. Despite the fact that a simple factorized form of the QDS-bath coupling Hamiltonian (24) was used to obtain Eqs. (57) and (58), they are valid for arbitrary (nonfactorized) forms of $\hat{W}(q)$.

For the following it is convenient to rewrite Eqs. (57) and (58) in terms of conventional (nongeneralized) functions. The resulting equations take the same form, but without instant source terms, which are replaced by the initial conditions at $t=0$, that is for $t>0$ one gets

$$
\begin{aligned}
\frac{d \sigma(t)}{d t}=\zeta(t)-i[\hat{H}, \sigma(t)]-i \int[\hat{W}(q), \eta(q, t)] d q & \\
\frac{\partial \eta(q, t)}{\partial t}= & -i[\hat{H}, \eta(q, t)]-i[\hat{W}(q), \sigma(t)]+[\hat{U}(q), \sigma(t)]_{+} \\
& +\left[\hat{Y}(q), i[\hat{H}, \sigma(t)]+\frac{d \sigma(t)}{d t}\right]_{+}+\mathcal{L}_{q} \eta(q, t),
\end{aligned}
$$

with the initial conditions,

$$
\sigma(t=0)=\sigma_{0}, \quad \eta(q, t=0)=\left[\hat{Y}(q), \sigma_{0}\right]_{+},
$$

where nonzero initial conditions for the auxiliary matrix $\eta$ arise from instant source term in Eq. (58), provided by the time derivative on its right-hand side. The initial conditions for auxiliary matrix accounts for the early $\left(t \leqslant \tau_{b}\right)$ nonMarkovian evolution, and their role is well demonstrated in Sec. VII. Note, however, that there also exists corresponding term in the right-hand side of Eq. (61). Thus, the account for the initial non-Markovian stage is reflected both in the "slipped" initial conditions and in the structure of the relaxation operator as well. For example, the Redfield theory is modified by using expansion (47) at $\tau \sim \tau_{c}$ in Eq. (14), which replaces Eq. (42) by

$$
\frac{d \sigma(t)}{d t}=-i[\hat{H}, \sigma(t)]-\left(1+\hat{\mathcal{R}}_{s}\right)^{-1} \hat{\mathcal{R}} \sigma(t), \quad t \gg \tau_{c},
$$

with the new "slipped" initial conditions,

$$
\sigma_{0} \rightarrow\left(1-\hat{\mathcal{R}}_{s}\right) \sigma_{0} .
$$

Here we have used conventional (nongeneralized functions), the relaxation operator $\hat{\mathcal{R}}$ is defined as before, see Eq. (42), while the "slippage" operator is of the form,

$$
\hat{\mathcal{R}}_{s}=-\int_{-\infty}^{\infty} \tau \hat{\mathcal{M}}(\tau) e^{-i \hat{\mathcal{H}} \tau} d \tau .
$$

Thus, both the initial conditions and the relaxation operator are modified if the early time evolution is accounted for, and, therefore, the problem cannot be reduced just to the "slipped" initial conditions.

The differential quantum-classical formulation in form of the two auxiliary local-time equations (60) and (61) utilizes approximations only with respect to the characteristic time $\tau_{b}$, but fully retains memory effects due to the dynamics of the classical degrees of freedom. In other words, it is not the same as Redfield theory, which is Markovian with respect to the entire memory effects. Needless to say, that the Redfield approach is more restrictive. On the other hand, our formulation provides an efficient quantum-classical treatment with a wide range of applicability and fully accounts for the memory effects due to the dynamics of the classical degrees of freedom without solution of the integrodifferential memory type equations.

The information about memory effects on the QDS evolution is coded in the matrix elements of the auxiliary matrix $\eta(t)$ [it cannot be called "auxiliary density matrix" since the trace of $\eta(t)$ is not conserved]. Note, however, that $\eta(t)$ contains information only about correlations specific to the 
given QDS-bath coupling, while the source term $\zeta(t)$ in Eq. (60) may reflect arbitrary types of initial correlations. The propagation of the QDS density matrix can be restarted at any time, provided that the matrix elements of $\eta$ are known.

The restriction of the time scale $\left(t \gg \tau_{b}\right)$, employed to obtain differential NQCA given by Eqs. (60)-(62), is not a serious limitation, since the bath degrees of freedom are treated classically. Strictly speaking, the classical description of the bath modes at very short times $\left(t \leqslant \tau_{b}\right)$ cannot be justified. In other words, the parameter $\tau_{b}$ determines the boundary between the time scales, when the bath behaves itself as a quantum-mechanical $\left[t \ll \hbar /\left(k_{B} T\right)\right]$ or a classical $\left[t \gg \hbar /\left(k_{B} T\right)\right]$ system. Thus, the NQCA implicitly assumes the applicability time scale $t \gg \tau_{b}$, and, in this sense, Eqs. (60)-(62) have the same applicability range as the nonMarkovian quantum-classical approximation in form of Eqs. (21), (39)-(40).

Although Eqs. (57) and (58) are local-time differential equations which can be directly solved in time domain without constructing/diagonalizing Liouville space operators, their practical applications are complicated by the necessity to solve the partial differential equation (58), which requires additional grids over phase space coordinates. Nevertheless, application of MD methods to model the bath dynamics resolves the problem, as it will be shown in subsequent article.

\section{NQCA AND BASIC PROPERTIES OF THE DENSITY MATRIX}

The differential NQCA in the form of the two auxiliary differential equations (60) and (61) preserves the basic properties of the density matrix. First of all, the trace of $\sigma(t)$ is conserved, since the traces from the commutator terms on the right-hand side of Eq. (60) are identically zero. The same is true for the trace of the time-extended source term $\zeta(t)$, as it is of a commutator form as well, see Eq. (15). Second, the Hermiticity of $\sigma(t)$ is also preserved, as it can be seen by taking the Hermitian conjugate of the equations and using the properties $(\hat{A} \hat{B})^{\dagger}=\hat{B}^{\dagger} \hat{A}^{\dagger}$ [operators $\hat{U}(q)$ and $\hat{Y}(q)$, defined in Eq. (59), are Hermitian].

The last required property, namely, the positive definiteness of the diagonal elements of $\sigma(t)$ (positivity) is difficult to prove in a general case. Nevertheless, for the simple example, considered in Sec. VII, the positivity breakdown has happened only when using parameter values outside the applicability range of the developed approach. This raises the question, whether the positivity breakdown can occur inside the applicability range of the differential NQCA, or not. In the latter case the more general integrodifferential formulation of NQCA, see Eqs. (21), (39)-(40), is likely to resolve the problem, but at the same time is more difficult for the numerical solution.

The properties discussed above must be fulfilled for any density matrix. The suggested NQCA additionally preserves the detailed balance principle, i.e., the Boltzmann equilibrium is established between all levels, involved in the relaxation. Indeed, the stationary solution of the Eqs. (60) and (61) requires $\zeta=\eta=0$ and

$$
[\hat{U}(q), \sigma]_{+}=i[\hat{W}(q), \sigma] .
$$

Substituting the QDS density matrix, corresponding to the Boltzmann equilibrium one, i.e., $\sigma=\exp (-\hat{H} / T)$, and using the definition of $\hat{U}(q)$, see Eq. (59), we get from Eq. (66) the following relation to prove

$$
\begin{gathered}
i \sum_{k, m} \tanh \left(\frac{\omega_{k m}}{2 T}\right) \hat{P}^{k} \hat{W}(q) \hat{P}^{m}\left(e^{-E_{m} / T}+e^{-E_{k} / T}\right) \\
=i \sum_{k, m} \hat{P}^{k} \hat{W}(q) \hat{P}^{m}\left(e^{-E_{m} / T}-e^{-E_{k} / T}\right),
\end{gathered}
$$

where we have also used the identity,

$$
\hat{W}(q) \equiv \sum_{k, m} \hat{P}^{k} \hat{W}(q) \hat{P}^{m} .
$$

Here $\hat{P}^{n}$ and $E_{n}$ are the projector operators on the eigenstates of the QDS Hamiltonian and the corresponding eigenvalues, respectively, see Eq. (33). Relation (67) holds for any term in the sum, i.e., at fixed indices $k, m$, since

$$
\frac{e^{-E_{m} / T}-e^{-E_{k} / T}}{e^{-E_{m} / T}+e^{-E_{k} / T}} \equiv \tanh \left(\frac{\omega_{k m}}{2 T}\right) .
$$

Thus, the Boltzmann equilibrium density matrix is indeed the stationary solution to Eqs. (60) and (61).

Using the semigroup approach originating from Lindblad's work, ${ }^{28}$ it has been shown that the Markovian master equation, which preserves positivity and satisfies the translational invariance, is incompatible with the approach to the canonical equilibrium state. ${ }^{29}$ This is well known fact in Redfield theory, which may violate the positivity of the density matrix for some extreme, but physically admissible initial conditions. ${ }^{4,30-32}$ It was also demonstrated, ${ }^{31}$ that the breakdown of the positivity in the Redfield master equation arises from the lack of memory effects in the early time evolution. In other words, at the initial non-Markovian stage of the density matrix evolution the initial conditions are mapped into those for which Markovian master equation preserves positivity. For the spin-boson model the problem can be overcome by construction of a "slippage" operator which prepares the "slipped" initial conditions for the Markovian master equation and incorporates the effect of the short-time evolution of the density matrix..$^{21,31}$ Nevertheless, there is no general proof that the "slipped" initial conditions preserve positivity of the Redfield theory. Moreover, a proper account for the short-time evolution of the density matrix leads to the modification of the relaxation operator as well, see Sec. V.

The problem of handling the initial correlations is well known in nonequilibrium statistical mechanics. ${ }^{33}$ It was shown, that for a wide class of dynamic systems in the thermodynamical limit there exists a so-called $\Pi$-operator of subdynamics, which projects correlations onto the two subspaces, invariant with respect to the time evolution. The time evolution in the П-subspace satisfies the Markovian master equation, which is exact on the entire time axis, while evolution in the complimentary subspace is of rather complicated character. ${ }^{33}$ The factorized initial state has commonly components in both subspaces, while the Markovian master equation description is valid only for particular initial correlations. The "slippage" operator ${ }^{21,31}$ can be considered as 
some analog of П-operator for the spin-boson model, projecting the initial state onto the subspace, which allows accurate description by the Markovian master equation.

Thus, at short times the description of the QDS evolution via local-time differential equations is, in general, not valid. The duration of this initial stage depends on the method in use, and is defined by $\tau_{b}$ for the suggested differential NQCA, while being correlation time $\tau_{c}$ of the bath for the Redfield theory. None the less, even if $\tau_{c}<\tau_{b}$ the description of the QDS evolution by the Markovian master equation provided by the Redfield theory is accurate only for the times $t \gg \tau_{b}$. Indeed, for a $\delta$-correlated bath the Fourier transform of the symmetrized correlation function $\widetilde{\mathcal{J}}(\omega)=$ const, but Eq. (39) still contains the memory kernel describing the decay of the quantum correlations on the time scale of $\tau_{b}$. Therefore, the assumption of a $\delta$-correlated bath is not sufficient to obtain a Markovian master equation in the case of finite temperature of the heat reservoir. The dynamical and the statistical properties of the bath are not independent, but related to each other. Increasing the temperature of the heat reservoir changes the equilibrium distribution over the bath states which, in turn, accelerates the dynamics of the bath and vice versa. In this sense, the assumption of a $\delta$-correlated bath implicitly assumes the infinite temperature of the bath, when $\tau_{b} \rightarrow 0$ and the Redfield master equation is of Lindblad form.

The factorized initial conditions for the differential NQCA eliminate the time-extended source term $\zeta(t)$ from Eq. (60). Nevertheless, at short times $t \sim \tau_{b}$ some specific correlations between the QDS and the bath are formed, which is reflected in the creation of nonzero elements of the auxiliary matrix $\eta(t)$. The suggested NQCA is automatically supplied by such "slipped" initial conditions, given by Eq. (62). The "slippage" consistently vanishes at $T \rightarrow \infty$ as the duration of the initial stage tends to zero in this limit. The role of the "slipped" initial conditions (62) is well demonstrated in Sec. VII, but anyway it is difficult to prove that they guarantee the positivity even within the applicability limits of the suggested NQCA.

The short-lifetime quantum correlations between the QDS and the bath play a decisive role for the establishment of the Boltzmann equilibrium (the detailed balance) between energy levels of the QDS in the course of time. The quantum phase, responsible for the dynamic transitions between energy levels, oscillates with the frequency corresponding to the energy difference. When this frequency $\omega_{k m}$ is small compared to the inverse quantum correlations lifetime $1 / \tau_{b}$ (or when $\omega_{k m} \tau_{b} \ll 1$ ), the QDS does not "feel" the quantum state of the bath, and the relaxation proceeds in the direction of level equilibration (in that case $\hat{U} \sim 0$ ). In the opposite situation $\omega_{k m} \tau_{b} \gtrsim 1$, however, phase evolves on a time scale comparable or shorter than $\tau_{b}$, and the transitions account for the quantum state of the bath, which results in detailed balance between the levels. For the canonical bath $\tau_{b}$ $\sim \hbar /\left(k_{B} T\right)$, which means equilibration between levels with $\Delta E /\left(k_{B} T\right) \ll 1$, and the detailed balance between levels with $\Delta E /\left(k_{B} T\right) \geqslant 1$. In other words, the information about quantum state of the bath is transferred into the QDS via their short-lifetime quantum correlations.

\section{TWO-LEVEL SYSTEM AND A SINGLE CORRELATION TIME BATH}

In order to test the suggested differential NQCA, we consider a simple case of a two-level quantum-dynamical subsystem, coupled to a single correlation time canonical bath. In that case $\mathcal{L}_{q}=1 / \tau_{c}$, and Eqs. (60) and (61) can be reduced to two ordinary differential equations,

$$
\begin{aligned}
\frac{d \sigma(t)}{d t}= & -i[\hat{H}, \sigma(t)]-i[\hat{V}, \eta(t)], \\
\frac{d \eta(t)}{d t}= & -i[\hat{H}, \eta(t)]-i[\hat{V}, \sigma(t)]+[\hat{U}, \sigma(t)]_{+} \\
& -i[\hat{Y},[\hat{V}, \eta(t)]]_{+}-\eta(t) / \tau_{c},
\end{aligned}
$$

with the initial conditions,

$$
\sigma(t=0)=\sigma_{0}, \quad \eta(t=0)=\left[\hat{Y}, \sigma_{0}\right]_{+} .
$$

Here, for the simplicity, we assumed that $\hat{W}(q)$ has factorized form (52), so that $\hat{U}$ and $\hat{Y}$ are given by Eqs. (49) and (50), respectively, and we also explicitely substituted time derivative in the right-hand side of Eq. (71).

Then, the Hamiltonian of the QDS and the QDS part of the coupling are chosen to be

$$
\hat{H}=\frac{1}{2}\left(\begin{array}{cc}
-\omega_{0} & 0 \\
0 & \omega_{0}
\end{array}\right), \quad \hat{V}=\frac{1}{2}\left(\begin{array}{cc}
0 & V_{0} \\
V_{0} & 0
\end{array}\right),
$$

and, therefore,

$$
\begin{aligned}
& \hat{U}=\frac{1}{2} \tanh \left(\frac{\omega_{0}}{2 T}\right)\left(\begin{array}{cc}
0 & -i V_{0} \\
i V_{0} & 0
\end{array}\right), \\
& \hat{Y}=\frac{1}{4 T} \frac{1}{\cosh ^{2}\left(\frac{\omega_{0}}{2 T}\right)}\left(\begin{array}{cc}
0 & V_{0} \\
V_{0} & 0
\end{array}\right) .
\end{aligned}
$$

First of all, we consider the case of zero splitting between levels $\left(\omega_{0}=0\right)$ and infinite temperature of the bath. In that case $\hat{H}=0, \hat{U}=\hat{Y}=0$ and the relaxation regimes are distinguished by the value of the dimensionless parameter $\alpha=V_{0} \tau_{c}$. There exists a simple analytical solution for the limiting case under consideration. It is of the form,

$$
n(t)=n_{0} e^{-t /\left(2 \tau_{c}\right)}\left(\cosh (\lambda t)+\frac{\sinh (\lambda t)}{2 \lambda \tau_{c}}\right),
$$

where

$$
\begin{aligned}
& n(t)=\sigma_{11}(t)-\sigma_{22}(t), \\
& \lambda=\frac{\sqrt{1-4 \alpha^{2}}}{2 \tau_{c}} \underset{4 \alpha^{2} \ll 1}{\simeq} \frac{1}{2 \tau_{c}}-V_{0}^{2} \tau_{c}, \quad \alpha=V_{0} \tau_{c} .
\end{aligned}
$$

Figures 3, 4 show the kinetics of the relaxation for three different values of the parameter $\alpha$, and the initial condition $n_{0}=1$. In the first case $\alpha=0.1$, which corresponds to the applicability range of Redfield theory [when the parameter $\lambda$, defined in Eq. (76), can be expanded], the kinetics has exponential character (with a relaxation rate equal to $V_{0}^{2} \tau_{c}$ $\left.=0.01 \tau_{c}\right)$, Fig. 3. In the second and the third cases $\alpha=1$ and 


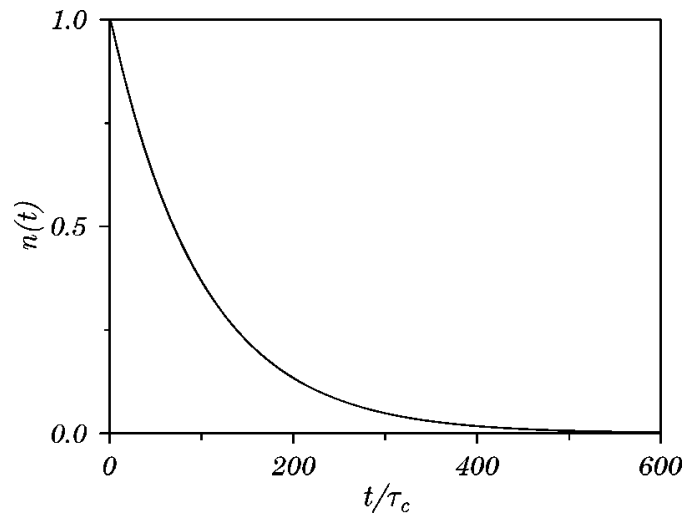

FIG. 3. Relaxation kinetics of the population difference for the applicability region of Redfield theory, $T=\infty, \omega_{0}=0, V_{0} \tau_{c}=0.1 \ll 1$, and $n_{0}=1$. The kinetics is exponential with the decay rate equal to $V_{0}^{2} \tau_{c}$.

$\alpha=10$, respectively, and Redfield theory cannot be applied. The parameter $\lambda$ becomes complex and the kinetics exhibits transient oscillations, while the relaxation time scale is independent of the QDS-bath coupling strength for $4 \alpha^{2}>1$ and is determined by the correlation time, see Fig. 4 .

Next we consider the situation where the splitting between levels is large enough to exhibit a considerable Boltzmann equilibrium population difference. Figure 5 shows the kinetics of the relaxation for $\tau_{c}=1.27 \mathrm{ps}, \omega_{0} \tau_{c}=100$, $V_{0} / \omega_{0}=0.2$, and different temperatures of the heat reservoir, indicated in the figure. Two stages in the relaxation kinetics are clearly seen, at the initial non-Markovian stage $t \leqslant \tau_{c}$ there appear transient oscillations, while on a longer time scale $t \gtrsim \tau_{c}$ the kinetics turns to be exponential. In the case of finite temperature of the bath the QDS relaxes to its canonical equilibrium state, while in the case of infinite temperature of the bath the levels are equilibrated in the course of relaxation. For $T=150$ the QDS-bath coupling strength satisfies $V_{0} \tau_{b} / 2=0.13 \ll 1$, and, therefore, the applicability criterion (44) is fulfilled.

It was pointed out several times, that the initial conditions of the form,

$$
\sigma_{11}=\sigma_{22}=\sigma_{12}=\sigma_{21}=1 / 2
$$

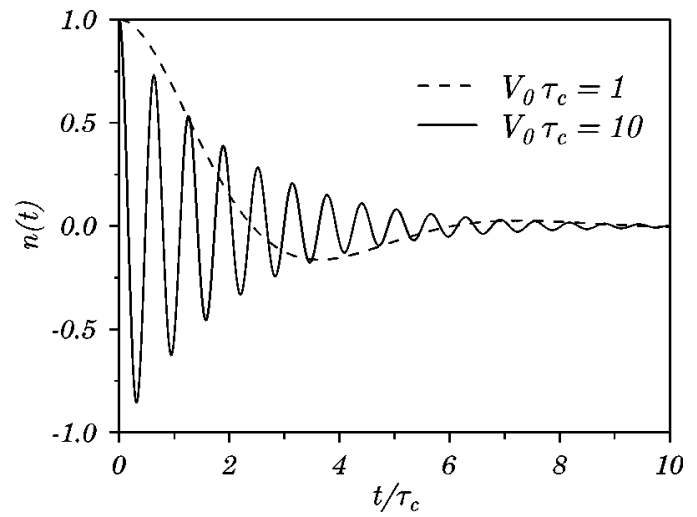

FIG. 4. Relaxation kinetics of the population difference beyond the applicability limits of Redfield theory. The parameters are: $T=\infty, \omega_{0}=0, n_{0}$ $=1$, and $V_{0} \tau_{c}=1$ (dashed line) or $V_{0} \tau_{c}=10$ (solid line).

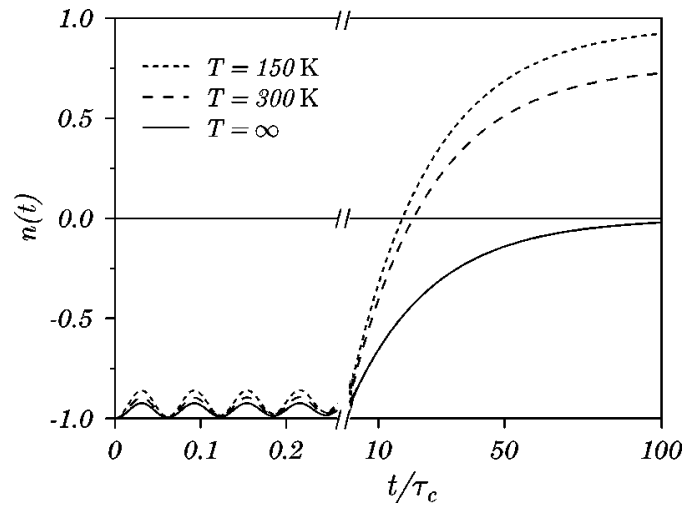

FIG. 5. Relaxation kinetics of the population difference in a weak coupling limit. The parameters are $\tau_{c}=1.27 \mathrm{ps}, \omega_{0} \tau_{c}=100, V_{0} / \omega_{0}=0.2$. The initial conditions for the density matrix are of the form $\sigma_{22}(0)=1, \sigma_{11}(0)$ $=\sigma_{12}(0)=0\left(n_{0}=-1\right)$, while the corresponding initial conditions for the auxiliary matrix $\eta$ are taken from Eq. (72). For the infinite temperature of the bath (solid line) relaxation proceeds in the direction of the levels equilibration, while in the case of finite temperature of the heat reservoir (dashed and long dashed lines) the QDS relaxes towards the canonical equilibrium state.

may serve as a good test of the quality of the quantumclassical equations. ${ }^{34,35}$ The attention in this case should be focused on the fundamental von Neumann condition for the density matrix elements,

$$
\sigma_{11} \sigma_{22} \geqslant\left|\sigma_{12}\right|^{2},
$$

which guarantees absence of negative eigenvalues of the density matrix of a two-level QDS (positivity). Figures 6-8 show the value,

$$
\gamma(t)=\frac{\left|\sigma_{12}(t)\right|^{2}}{\sigma_{11}(t) \sigma_{22}(t)}
$$

as a function of time, and the condition (78) means 0 $\leqslant \gamma(t) \leqslant 1$. Figures 6 and 7 demonstrate the role of the initial conditions (72) for the auxiliary matrix $\eta$. The initial conditions for the $\sigma$ are taken from Eq. (77), while the elements of

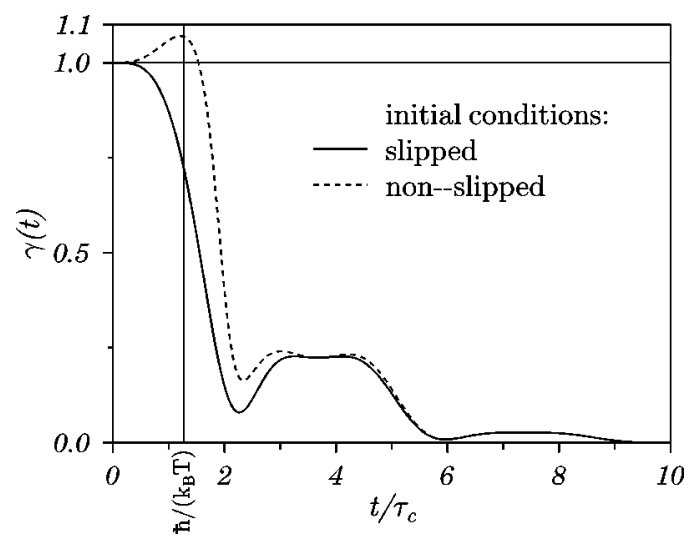

FIG. 6. Demonstration of the effect of the initial conditions for the auxiliary matrix $\eta$. Time evolution of the parameter $\gamma(t)$, see Eq. (78), is shown, $\gamma$ $>1$ and $\gamma<0$ indicate positivity breakdown. Solid line corresponds to the "slipped" initial conditions, defined by Eq. (72), while dashed line corresponds to a zero auxiliary matrix at the initial instance of time (neglect of the memory effects from the earlier time evolution). The parameters are $\omega_{0}=10^{14} \mathrm{rad} / \mathrm{s}, V_{0}=1.5 \omega_{0}, \tau_{c}=10^{-14} \mathrm{~s}, T=300 \mathrm{~K}$. The initial conditions for the density matrix are taken from Eq. (77). 


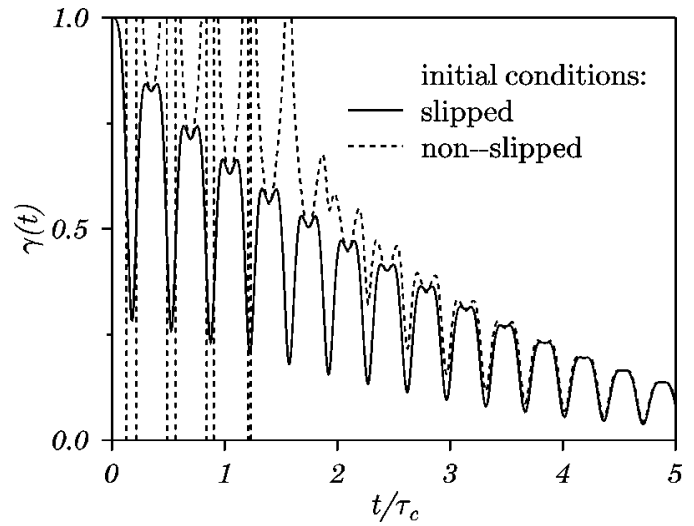

FIG. 7. Same as Fig. 6, but with 10 times longer correlation time, i.e., $\tau_{c}$ $=10^{-13} \mathrm{~s}$.

the auxiliary matrix at the initial instant of time are determined either from Eq. (72) ("slipped" initial conditions) or set to zero. In the former case (solid line) $0 \leqslant \gamma(t) \leqslant 1$, while in the latter case (dashed line) the positivity is violated, see Figs. 6 and 7. The parameter values are indicated on the figure captions. Thus, the neglect of the memory effects in the early time evolution $\left(t \lesssim \tau_{b}\right)$ may indeed lead to the positivity breakdown.

In the simple cases, considered above, the NQCA with a "slipped" initial conditions (72) preserved the positivity. Nevertheless, the suggested NQCA does not preserve positivity by construction, unlike the Lindblad master equation. Figure 8 shows the time evolution of the parameter $\gamma(t)$ increasing the QDS-bath coupling strength $V_{0} / 2$. It is readily seen, that the "slipped" initial conditions (72) preserve positivity only within the applicability range (44) of the developed approach, which for the model under consideration is $V_{0} \tau_{b} / 2 \ll 1$.

\section{CONCLUSION}

A quantum-classical approximation, capable to describe the evolution of quantum-dynamical subsystem coupled with dissipative environment well beyond the applicability limits of Redfield theory was suggested. It fully accounts for the arbitrary long memory of the heat reservoir, but is of differential form. The theory utilizes the fast decay of the crosscorrelations between the quantum-dynamical subsystem and the bath provided by the energy dispersion of the bath degrees of freedom. This lifetime is proportional to the inverse temperature of the heat reservoir (at room temperature it is 8 fs) and allows to study various relaxation processes since a subpicosecond time range. The short-lifetime "quantum" correlations, however, are responsible for the establishment of the canonical equilibrium (the detailed balance) between energy levels of open quantum system, and are taken into account.

In recent years there has been an extensive discussion of the different approaches to the quantum dissipation in connection to the Lindblad master equation, see Ref. 36. We have shown, that for the case of finite temperature of the heat reservoir, a master equation for the reduced density matrix becomes non-Markovian even for a $\delta$-correlated bath. Due to

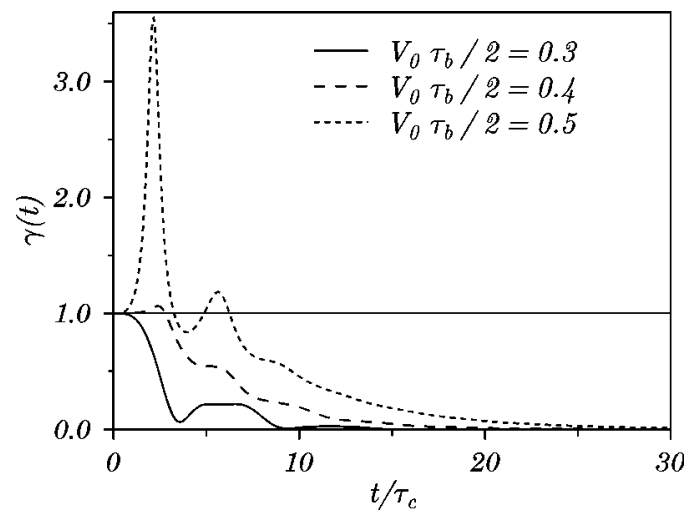

FIG. 8. Demonstration of the positivity breakdown outside applicability limits of the developed approach. All parameter values are the same as for Fig. 6, except the values of the QDS-bath coupling strength $V_{0} / 2$, indicated in the figure ( $\left.\tau_{b}=8 \mathrm{fs}\right)$.

a principal impossibility of Markovian description at times $t \lesssim \hbar /\left(k_{B} T\right)$ the differential master equation approaches may lead to the positivity breakdown for some extreme but physically allowed initial conditions. Hence, the Lindblad form of master equation, which preserves positivity by construction, is not adequate for the case of finite temperature of the bath.

The use of differential description on the time scale, larger than the lifetime of the quantum correlations, requires a proper account for an early time evolution to preserve positivity of the density matrix, and the developed approach is automatically supplied by such a "slipped" initial conditions. We also suggested the corresponding improvement of the Redfield theory, where both the initial conditions and the relaxation operator are modified. Simple example of a twolevel quantum subsystem coupled to a single correlation time canonical bath well served to explore capabilities of the developed method, while applications to multilevel systems of practical interest together with molecular dynamics technique to simulate the bath dynamics are considered in the subsequent article.

\section{ACKNOWLEDGMENT}

The author is grateful to the Alexander von Humboldt Foundation for a postdoctoral fellowship and to Professor Jürgen Troe for continued support of this work.

\section{APPENDIX A: LIOUVILLE SPACE FORMULATION}

The Liouville-von Neumann equation for the density matrix (3) can formally be written in a form similar to the Schrödinger equation in several ways. First, one can introduce a vector with $N^{2}$ ( $N$ is the number of the states, included into consideration) elements, composed of the matrix elements of the density matrix. Then the operators in the right-hand side of Eq. (4) become regular linear operators (matrices) in the Hilbert space, and with the dimensionality $N^{2} \times N^{2}$. This approach is widely used in numerical calculations. Second, one can use the so-called Liouville space operators (superoperators). In the latter case a matrix algebra is simply replaced by a tensor algebra, i.e., operators in Eq. (4) become tensors. 
For our purposes we use Liouville operators in the basis of direct product between QDS and bath states. Matrix elements between QDS states are denoted by the lower Latin indices, while matrix elements between bath states are denoted by the upper Greek letters. Thus, the Liouville operators are tensors of rank 8, and their matrix elements are expressed via matrix elements of the corresponding Hilbert space operators by the following formulas:

$$
\begin{aligned}
& \mathcal{H}_{i k ; l m}^{\alpha \beta ; \mu \nu}=H_{m k}^{\nu \beta} \delta_{i l} \delta_{\alpha \mu}-H_{i l}^{\alpha \mu} \delta_{k m} \delta_{\beta \nu}, \\
& \mathcal{F}_{i k ; l m}^{\alpha \beta ; \mu \nu}=F_{m k}^{\nu \beta} \delta_{i l} \delta_{\alpha \mu}-F_{i l}^{\alpha \mu} \delta_{k m} \delta_{\beta \nu}, \\
& \mathcal{W}_{i k ; l m}^{\alpha \beta ; \mu \nu}=W_{m k}^{\nu \beta} \delta_{i l} \delta_{\alpha \mu}-W_{i l}^{\alpha \mu} \delta_{k m} \delta_{\beta \nu},
\end{aligned}
$$

where

$$
\delta_{i j}= \begin{cases}1 & i=j, \\ 0 & i \neq j,\end{cases}
$$

is the Kronecker delta. The QDS Hamiltonian $\hat{H}$ has only diagonal matrix elements between bath states, while the bath Hamiltonian $\hat{F}$ is diagonal between QDS states, i.e.,

$$
H_{m k}^{\nu \beta}=H_{m k} \delta_{\nu \beta}, \quad F_{m k}^{\nu \beta}=F^{\nu \beta} \delta_{m k} .
$$

The product of two Liouville operators is defined as

$$
(\hat{\mathcal{A}} \hat{\mathcal{B}})_{i k ; l m}^{\alpha \beta ; \mu \nu}=\sum_{j, n ; \xi \eta} \mathcal{A}_{i k ; j n}^{\alpha \beta ; \xi \eta} \mathcal{B}_{j n ; l m}^{\xi \eta ; \mu \nu}
$$

while the operation of the Liouville operator on the density matrix is given by

$$
(\hat{A} \varrho)_{i k}^{\alpha \beta}=\sum_{l, m ; \mu \nu} \mathcal{A}_{i k ; l m}^{\alpha \beta ; \mu \nu} \varrho_{l m}^{\mu \nu} .
$$

Unity operator $\hat{1}$ is defined as usual,

$$
\hat{\mathcal{A}} \hat{1}=\hat{1} \hat{\mathcal{A}}=\hat{\mathcal{A}}, \quad 1_{i k ; l m}^{\alpha \beta ; \mu \nu}=\delta_{i l} \delta_{k m} \delta_{\alpha \mu} \delta_{\beta \nu} .
$$

It can easily be seen, that for the Liouville operators, defined by Eqs. (A1)-(A3) one has

$$
i \hat{\mathcal{W}} \varrho=-i[\hat{W}, \varrho] \text {, }
$$

i.e., they are "commutator derived superoperators." Indeed, from Eqs. (A3) and (A7) one has

$$
\begin{aligned}
i(\hat{\mathcal{W}} \varrho)_{i k}^{\alpha \beta} & =i \sum_{l, m ; \mu \nu}\left(W_{m k}^{\nu \beta} \delta_{i l} \delta_{\alpha \mu}-W_{i l}^{\alpha \mu} \delta_{k m} \delta_{\beta \nu}\right) \varrho_{l m}^{\mu \nu} \\
& =i \sum_{m ; \nu} \varrho_{i m}^{\alpha \nu} W_{m k}^{\nu \beta}-i \sum_{k ; \mu} W_{i l}^{\alpha \mu} \varrho_{l k}^{\mu \beta} \\
& =-i([\hat{W}, \varrho])_{i k}^{\alpha \beta} .
\end{aligned}
$$

The advantage of taking the Liouville operators in the basis of the direct product is the simplicity of taking the trace over the QDS or the bath subsystems. For example,

$$
\operatorname{Tr}_{b}(\hat{\mathcal{A}} \varrho)=\sum_{\alpha} \sum_{l, m ; \mu \nu} \hat{\mathcal{A}}_{i k ; l m}^{\alpha \alpha ; \mu \nu} \varrho_{l m}^{\mu \nu}
$$

is a simplification of the tensor over first two upper indices (for the trace over QDS the simplification is carried out over first two lower Latin indices).
The commutator derived superoperators (A1)-(A3) have an important property, the trace over all states yields zero. (For QDS Liouvillian $\hat{\mathcal{H}}$ it is sufficient to take trace over the QDS states only, while for the bath Liouvillian $\hat{\mathcal{F}}$-only over the bath states), i.e.,

$$
\operatorname{Tr}(\hat{\mathcal{W}} \varrho)=0, \quad \operatorname{Tr}_{q}(\hat{\mathcal{H}} \varrho)=0, \quad \operatorname{Tr}_{b}(\hat{\mathcal{F}} \varrho)=0
$$

It can be proved directly, using the explicit expressions for the matrix elements (A1)-(A3), and reflects the particle conservation law by the Liouville-von Neumann equation.

Finally we give useful expression for the free evolution operator $\hat{\mathcal{G}}_{0}(t)$, defined by Eq. (11). Its operation on the density matrix,

$$
\hat{\mathcal{G}}_{0}(t) \varrho=\theta(t) e^{-i \hat{F} t} e^{-i \hat{H} t} \varrho e^{i \hat{H} t} e^{i \hat{F} t} .
$$

For the proof of Eq. (A13) it is sufficient to note, that the Hamiltonians (and Liouvillians) of the QDS and the bath commute with each other, i.e.,

$$
\hat{\mathcal{G}}_{0}(t)=\hat{\mathcal{T}}_{0}(t) \times e^{i \hat{\mathcal{F}} t}, \quad \hat{\mathcal{T}}_{0}(t)=\theta(t) e^{i \hat{\mathcal{H}} t},
$$

and use the basis of the direct product of the eigenstates of the QDS and the bath Hamiltonians, i.e.,

$$
|\psi\rangle_{n}^{\alpha}=|n\rangle|\alpha\rangle, \quad \hat{H}|n\rangle=E_{n}|n\rangle, \quad \hat{F}|\alpha\rangle=\mathcal{E}_{\alpha}|\alpha\rangle,
$$

where $E_{n}$ and $\mathcal{E}_{\alpha}$ are the corresponding eigenvalues. Then we get

$$
\begin{aligned}
\left(\hat{\mathcal{G}}_{0}(t) \varrho\right)_{i k}^{\alpha \beta} & =\theta(t) e^{-i\left(\mathcal{E}_{\alpha}-\mathcal{E}_{\beta}\right) t} e^{-i\left(E_{i}-E_{k}\right) t} \varrho_{i k}^{\alpha \beta} \\
& =\theta(t)\left(e^{-i \hat{F} t} e^{-i \hat{H} t} \varrho e^{i \hat{H} t} e^{i \hat{F} t}\right)_{i k}^{\alpha \beta} .
\end{aligned}
$$

In principle, Eqs. (A9) and (A13) are sufficient to convert all Liouville space expressions, derived in the current article, into the Hilbert space representation.

\section{APPENDIX B: QUANTUM CORRELATION FUNCTIONS AND RELATIONS BETWEEN THEM}

Using the basis of the eigenstates of the bath Hamiltonian, the quantum correlation functions, defined by Eqs. (26), can be written as

$$
\begin{aligned}
& j_{1}(\tau)=\frac{1}{Z_{b}} \sum_{\alpha, \beta} B^{\alpha \beta} B^{\beta \alpha} e^{i \nu_{\alpha \beta} \tau} e^{-\mathcal{E}_{\alpha} / T}, \\
& j_{2}(\tau)=\frac{1}{Z_{b}} \sum_{\alpha, \beta} B^{\alpha \beta} B^{\beta \alpha} e^{i \nu_{\alpha \beta} \tau} e^{-\mathcal{E}_{\beta} / T},
\end{aligned}
$$

where

$$
\nu_{\alpha \beta}=\mathcal{E}_{\alpha}-\mathcal{E}_{\beta} .
$$

For the proof of Eq. (28) it is sufficient to change the summation indices $(\alpha, \beta) \rightarrow(\beta, \alpha)$ in the expression for $j_{2}$ $(-\tau)$, given by the second equation in Eq. (B1) after inversion of the time, since $\nu_{\beta \alpha} \equiv-\nu_{\alpha \beta}$.

The relation between Fourier transforms of quantum correlation functions, see Eq. (29), is proved as follows. Fourier transforms of $j_{1,2}(30)$, and with account for Eqs. (B1) give 


$$
\begin{aligned}
& \tilde{j}_{1}(\omega)=\frac{1}{Z_{b}} \sum_{\alpha, \beta} B^{\alpha \beta} B^{\beta \alpha} \delta\left(\omega-\nu_{\alpha \beta}\right) e^{-\mathcal{E}_{\alpha} / T}, \\
& \tilde{j}_{2}(\omega)=\frac{1}{Z_{b}} \sum_{\alpha, \beta} B^{\alpha \beta} B^{\beta \alpha} \delta\left(\omega-\nu_{\alpha \beta}\right) e^{-\mathcal{E}_{\beta} / T} .
\end{aligned}
$$

Then we use the following identity:

$$
\begin{aligned}
\delta\left(\omega-\nu_{\alpha \beta}\right) e^{-\mathcal{E}_{\beta} / T} & \equiv \delta\left(\omega-\nu_{\alpha \beta}\right) e^{\nu_{\alpha \beta} / T} e^{-\mathcal{E}_{\alpha} / T} \\
& \equiv e^{\omega / T} \delta\left(\omega-\nu_{\alpha \beta}\right) e^{-\mathcal{E}_{\alpha} / T}
\end{aligned}
$$

in Eq. (B3) for $\tilde{j}_{2}(\omega)$ to get Eq. (29).

\section{APPENDIX C: EQUIVALENCE OF DIFFERENTIAL AND INTEGRODIFFERENTIAL FORMS OF QUANTUM-CLASSICAL APPROXIMATION}

The equivalence of Eqs. (21), (51) and Eqs. (57)-(58) is proved as follows. The Fourier transform of Eqs. (57) and (58) gives

$$
\begin{aligned}
i \omega \widetilde{\sigma}(\omega)+i[\hat{H}, \widetilde{\sigma}(\omega)]= & \sigma_{0}+\widetilde{\zeta}(\omega) \\
& -\int i[\hat{W}(q), \widetilde{\eta}(q, \omega)] d q,
\end{aligned}
$$

$$
i \omega \widetilde{\eta}(q, \omega)+i[\hat{H}, \widetilde{\eta}(q, \omega)]-\mathcal{L}_{q} \widetilde{\eta}(q, \omega)=\sigma_{x}(q, \omega),
$$

where we introduced the notation,

$$
\begin{aligned}
\sigma_{x}(q, \omega) \equiv & -i[\hat{W}(q), \widetilde{\sigma}(\omega)]+[\hat{U}(q), \widetilde{\sigma}(\omega)]_{+} \\
& +[\hat{Y}(q), i[\hat{H}, \widetilde{\sigma}(\omega)]+i \omega \widetilde{\sigma}(\omega)]_{+} .
\end{aligned}
$$

Then we introduce the resolvent operator $\hat{\mathcal{G}}_{f}\left(q, q_{0} ; \omega\right)$,

$$
\left(i \omega-i \hat{\mathcal{H}}-\mathcal{L}_{q}\right) \hat{\mathcal{G}}_{f}\left(q, q_{0} ; \omega\right)=\delta\left(q-q_{0}\right),
$$

which can be represented as

$$
\hat{\mathcal{G}}_{f}\left(q, q_{0} ; \omega\right)=\int_{-\infty}^{\infty} \overline{\mathcal{P}}\left(q, q_{0} ; t\right) e^{i \hat{\mathcal{H}} t} e^{-i \omega t} d t,
$$

where $\overline{\mathcal{P}}\left(q, q_{0} ; t\right)$ satisfies Eq. (55), and write the formal solution of Eq. (C2) as

$$
\widetilde{\eta}(q, \omega)=\int \hat{\widetilde{\mathcal{G}}}_{f}\left(q, q_{0} ; \omega\right) \sigma_{x}\left(q_{0}, \omega\right) d q_{0} .
$$

Finally, we use in Eq. (C6) the identity

$$
\begin{aligned}
\hat{\mathcal{G}}_{f}\left(q, q_{0} ; \omega\right) \sigma_{x}\left(q_{0}, \omega\right) \\
\quad=\sum_{k, m} \widetilde{\mathcal{P}}\left(q, q_{0} ; \omega+\omega_{k m}\right) \hat{P}^{k} \sigma_{x}\left(q_{0}, \omega\right) \hat{P}^{m},
\end{aligned}
$$

assume the bilinear form of QDS-bath coupling (52) and, substituting the resulting expression in Eq. (C1), we get Eqs. (21) and (51), where the retarded correlation function is identified with the classical one, defined by Eqs. (53) and (55).

${ }^{1}$ A. G. Redfield, IBM J. Res. Dev. 1, 19 (1957); A. G. Redfield, Adv. Magn. Reson. 1, 1 (1965).

${ }^{2}$ K. Blum, Density Matrix Theory and Applications (Plenum, New York, 1981).

${ }^{3}$ C. P. Slichter, Principles of Magnetic Resonance (Springer, Berlin, 1990).

${ }^{4}$ N. G. van Kampen, Stochastic Processes in Physics and Chemistry (North-Holland, Amsterdam, 1992).

${ }^{5}$ W. T. Pollard and R. A. Friesner, J. Chem. Phys. 100, 5054 (1994).

${ }^{6}$ Y. Tanimura and P. G. Wolynes, Phys. Rev. A 43, 4131 (1991); Y. Tanimura and P. G. Wolynes, J. Chem. Phys. 96, 8485 (1992).

${ }^{7}$ J. Cao, J. Chem. Phys. 107, 3204 (1997).

${ }^{8}$ C. Meier and D. J. Tannor, J. Chem. Phys. 111, 3365 (1999).

${ }^{9}$ H. P. Breuer, D. Faller, B. Kappler, and F. Petruccione, Phys. Rev. A 60, 3188 (1999)

${ }^{10}$ J. Wilkie, J. Chem. Phys. 114, 7736 (2001).

${ }^{11}$ T. Mančal and V. May, J. Chem. Phys. 114, 1510 (2001).

${ }^{12}$ S. Jang, J. Cao, and R. J. Silbey, J. Chem. Phys. 116, 2705 (2002).

${ }^{13}$ E. Neria and A. Nitzan, J. Chem. Phys. 99, 1109 (1993); S. A. Egorov, E. Rabani, and B. J. Berne, J. Phys. Chem. 103, 10978 (1999).

${ }^{14}$ K. Thompson and N. Makri, J. Chem. Phys. 110, 1343 (1999).

${ }^{15}$ J. C. Tully and R. K. Preston, J. Chem. Phys. 55, 562 (1971); F. Webster, J. Schnitker, M. S. Friedrich, R. A. Friesner, and P. J. Rossky, Phys. Rev. Lett. 66, 3172 (1991).

${ }^{16}$ P. A. M. Dirac, Proc. Cambridge Philos. Soc. 26, 376 (1930).

${ }^{17}$ N. Makri, Annu. Rev. Phys. Chem. 50, 167 (1999), and references therein.

${ }^{18}$ R. Car and M. Parrinello, Phys. Rev. Lett. 55, 2471 (1985).

${ }^{19}$ E. J. Heller, J. Chem. Phys. 62, 1544 (1975); 75, 2923 (1981).

${ }^{20}$ H.-P. Breuer, B. Kappler, and F. Petruccione, Phys. Rev. A 59, 1633 (1999).

${ }^{21}$ P. Gaspard and M. Nagaoka, J. Chem. Phys. 111, 5668 (1999).

${ }^{22}$ U. Fano, Phys. Rev. 131, 259 (1963).

${ }^{23}$ I. M. Gelfand and G. E. Shilov, Generalized Functions (Academic, New York, 1964); R. P. Kanwal, Generalized Functions: Theory and Technique (Springer, Berlin, 1998).

${ }^{24}$ S. Nakajima, Prog. Theor. Phys. 20, 948 (1958); R. Zwanzig, J. Chem. Phys. 33, 1338 (1960).

${ }^{25}$ B. J. Berne, Time-Dependent Properties of Condensed Media, Vol. 8B, in Physical Chemistry: An Advanced Treatise (Academic, New York, 1971), p. 539.

${ }^{26}$ U. Weiss, Quantum Dissipative Systems (World Scientific, Singapore, 1999).

${ }^{27}$ D. V. Oxtoby, Adv. Chem. Phys. 47, 487 (1981).

${ }^{28}$ G. Lindblad, Commun. Math. Phys. 48, 119 (1976).

${ }^{29}$ G. Lindblad, Rep. Math. Phys. 10, 393 (1976).

${ }^{30} \mathrm{R}$. Alicki and K. Lendi, Quantum Dynamical Semigroups and Applications (Springer, Berlin, 1987).

${ }^{31}$ A. Suarez, R. Silbey, and I. Oppenheim, J. Chem. Phys. 97, 5101 (1992).

${ }^{32}$ P. Pechukas, Phys. Rev. Lett. 73, 1060 (1994).

${ }^{33} \mathrm{R}$. Balescu, Equilibrium and Nonequilibrium Statistical Mechanics (Wiley, New York, 1975).

${ }^{34}$ P. A. Frantsuzov, Chem. Phys. Lett. 267, 427 (1997)

${ }^{35}$ M. V. Basilevsky, A. V. Soudackov, and A. I. Voronin, Chem. Phys. 235 , 281 (1998).

${ }^{36}$ D. Kohen, C. C. Marston, and D. J. Tannor, J. Chem. Phys. 107, 5236 (1997), and references therein. 